\title{
Identifying Performance Outliers for Stroke Care Based on Composite Score of Process Indicators: an Observational Study in China
}

\author{
Chao Wang, $M D^{7}$, Shaofei Su, MD², Xi Li, $M D^{7}$, Jingkun $L i, M D^{7}$, Xiaoqiang Bao, $M D^{7}$, and \\ Meina Liu, MD ${ }^{7}$
}

'Department of Biostatistics, Public Health College, Harbin Medical University Harbin, China; ${ }^{2}$ Central Laboratory, Beijing Obstetrics and Gynecology Hospital, Capital Medical University Chaoyang, Beijing, China.

\begin{abstract}
BACKGROUND: Variability in the quality of stroke care is widespread. Identifying performance-based outlier hospitals based on quality indicators (QIs) has become a common practice.

OBJECTIVES: To develop a tool for identifying performance-based outlier hospitals based on riskadjusted adherence rates of process indicators.

DESIGN: Hospitals were classified into five-level outliers based on the observed-to-expected ratio and $P$ value. The composite quality score was derived by summation of the points for each indicator for each hospital, and associations between outlier status and outcomes were determined.
\end{abstract}

PARTICIPANTS: Patients diagnosed with acute ischemic stroke, January 1, 2011-May 31, 2017.

INTERVENTION: N/A

MAIN OUTCOME MEASURES: Independence at discharge (the modified Rankin Scale $=0-2$ ).

KEY RESULTS: A total of 501,132 patients from 519 hospitals were identified. From 0.39 to $19.65 \%$ of hospitals were identified as high outliers according to various QIs. Composite quality scores ranged from -20 to 16 . Providers that were high outliers based on QI2, QI8, QI9, and QI11 had higher independent rates. For composite quality score, each point increase corresponded to an $8 \%$ increase in the odds of independent rate.

CONCLUSION: Nationwide variation in the quality of acute stroke care exists at the hospital level. Variability in the quality of stroke care can be captured by our proposed quality score. Applying this quality score as a benchmarking tool could provide audit-level feedback to policymakers and hospitals to aid quality improvement.

KEY WORDS: stroke; quality of care; composite indicator; performance measures; outliers.

J Gen Intern Med 35(9):2621-8

DOI: $10.1007 / \mathrm{s} 11606-020-05923-\mathrm{x}$

(C) Society of General Internal Medicine 2020

Electronic supplementary material The online version of this article (https://doi.org/10.1007/s11606-020-05923-x) contains supplementary material which is available to authorized users.

Received November 9, 2019

Accepted May 11, 2020

Published online May 27, 2020

\section{BACKGROUND}

Stroke is a global health problem, with 10.3 million new cases of stroke accounting for 113 million disability-adjusted life years (DALYs) per year. ${ }^{1}$ Although stroke treatment has evolved, the quality of stroke care varies globally, and inequalities exist between medical facilities even within the same country. ${ }^{2,3}$ Comparing health care providers and identifying outlier providers simultaneously based on various indicators of performance, such as the rate of occurrence of particular outcome or process-based measures, has become a common practice. ${ }^{4-6}$

Outcomes-based quality indicators are by far the most common measures in quality performance comparisons and identification of hospital outliers. ${ }^{7,8}$ Outlier hospitals can be identified simply according to relative rank based on expected mortality (after risk-adjusted) (e.g., top 10\%, bottom 10\%) or by identifying hospitals that are statistically significantly different from a given benchmark or standard (commonly the overall average mortality in the population being evaluated). ${ }^{7}$ Observed-to-expected $(\mathrm{O} / \mathrm{E})$ ratio is a representative method, which consider both the observed and expected rates, and combine the statistical significance to classify hospitals' performance $^{7,9,10}$. However, for outcomes measures, reliability is susceptible to small sample size and low incidence rates, and it can be challenging to obtain granular data allowing adequate risk adjustment that accurately reflects the quality of care a patient actually received. ${ }^{11,12}$ The challenge of these quality improvement metrics after identifying outliers based on outcome is whether they can be modified by alterations in care processes and consequently improved upon. As a result, outcomes may be less actionable.

Process-based quality indicators are also important part in evaluation of quality of care. The standardization and integrity of clinical therapy has important impacts on patients' outcome. There is evidence that focusing on process measures such as increased adherence to evidence-based guidelines can improve stroke treatment and prognosis in Sweden. ${ }^{13}$ One recent study based on data from global health systems found that improvements in the quality of health services would have a greater overall effect on mortality than expansion of service coverage. ${ }^{14}$ In comparison, process-based indicators are 
readily modifiable and can potentially offer providers definable targets for improvement. ${ }^{10}$ However, discriminating the quality of care using process indicators also involves a number of challenges. Treatment processes are influenced by many factors, including patient-level and provider-level characteristics. It is also necessary to adjust patient case mix for the process measures. Another major hurdle is that the multidisciplinary nature of treatment makes it difficult to use a single process indicator for evaluation. Combining individual indicators into a composite performance measure is a common strategy. There are many approaches to calculate the composite quality score, such as equal-weights, denominator-based weights, numerator-based weights, all-or-none scoring measure, and quality score cutoff measure. ${ }^{15,}{ }^{16}$ Each method inevitably has some issues.

For acute stroke, previous measures based on outcomes indicator may not be able to well capture the hospital-level quality variation, when it is difficult to acquire the patients' long-term outcomes. ${ }^{17,18}$ Additionally, some researchers suggested that process measure should also be risk-adjusted. ${ }^{19,} 20$ In this study, we sought to develop a tool for identifying performance-based outlier hospitals based on risk-adjusted adherence rates of stroke process indicators. It helps discover hospitals with higher or lower performance than the expected after case-mix adjustment. The composite performance comprehensively considered the outlier status of hospitals on each indicator, exploring the relationship between a composite quality score and outcomes. Such an analysis method could enable high-performing hospitals to maintain best practices and allow underperformers to understand how much benefit they would receive from quality improvement efforts, providing motivation to critically appraise their multidisciplinary care processes.

\section{METHODS}

\section{Data Source and Study Population}

We obtained data on patients with acute ischemic stroke from the Medical Care Quality Management and Control System for Specific Disease of China. The system is designed to collect and manage data on quality indicators (QIs) within the health care system. All registered hospitals submitted data by clinical teams via a web-based portal with real-time data validation. To minimize error caused by manual reporting, the information of the front page of inpatient medical records was collected automatically. Data used in the study were fully anonymized. Because the data were collected for administration of quality of care rather than research, ethical approval was not required under China research governance arrangements. The requirement for informed consent was waived as it was determined that this study does not meet the regulatory definition of human subject research.

Hospital-level data and patient-level data were combined according to hospital codes. A cohort of patients with acute ischemic stroke diagnosed from January 1, 2011, to May 31, 2017, was identified by the International Classification of Diseases, 10th revision (ICD)-10 codes. The exclusion criteria are shown in Figure 1. Patients transferred to another hospital were first excluded, followed by hospitals with fewer than 20 admissions overall and missing information to identify denominators of selected QIs in the study. Finally, the analytical population comprised 501,132 patients who received inpatient treatment from 519 hospitals.

\section{Qls and Outcomes}

QIs for stroke care were established by the National Health Commission of the People's Republic of China in 2009, which organized a national multidisciplinary board included the clinical and hospital management experts. The establishing process of indicators was based on a systematic literature review, international and Chinese recommendations. Most of the quality indicators were developed and adapted from the USA. Because medication information after discharge was difficult to obtain, some indicators about drug treatment post-discharge were not included in the study. We used 13 process indicators in this analysis. The indicators are listed in Supplement Table 1.

The functional outcomes at discharge of stroke patients were assessed with the modified Rankin Scale (mRS) score at discharge. ${ }^{21}$ The mRS is a commonly used scale for measuring the degree of disability or dependence in the daily activities of people who have suffered a stroke or other causes of neurological disability. It has become the most widely used clinical outcome measure for stroke. ${ }^{7,22}$ Patient outcome was classified as independence (mRS score 0-2) or disability/death (mRS score 3-6). ${ }^{23,24}$ The primary outcome of this study was the independent rate, which was defined as the proportion of patients discharged with mRS score 0 to 2 .

\section{Variables}

Patient-level covariates included year and age at diagnosis $(\leq$ $55,55-65,65-75$, and $>75$ ); primary payer status (socialized medicine, basic medical insurance, commercial medical insurance, and self-paying); pattern of admission (outpatient, emergency); National Institutes of Health Stroke Scale (NIHSS) score on arrival; and comorbidities identified by the ICD-10 codes including diabetes mellitus, myocardial infarction, pneumonia, coronary heart disease, renal failure, chronic obstructive pulmonary disease, arrhythmia, hematological diseases, cerebrovascular diseases, rheumatic valvular heart disease, and congestive heart failure. In China, the insurance reimbursement rates of socialized medicine, basic medical insurance, and commercial medical insurance are 85-95\%, $50-65 \%$, and $75 \%$, respectively. We classified the stroke severity into four categories: no stroke symptoms (NIHSS = 0 ), minor stroke (NIHSS $=1-4$ ), moderate to severe stroke (NIHSS $=16-20)$, and severe stroke (NIHSS $=21-42) .{ }^{25} \mathrm{In}$ our study, anemia was included in hematological diseases. 
A total of 571,522 patients (treated in 755 hospitals) with acute ischemic stroke

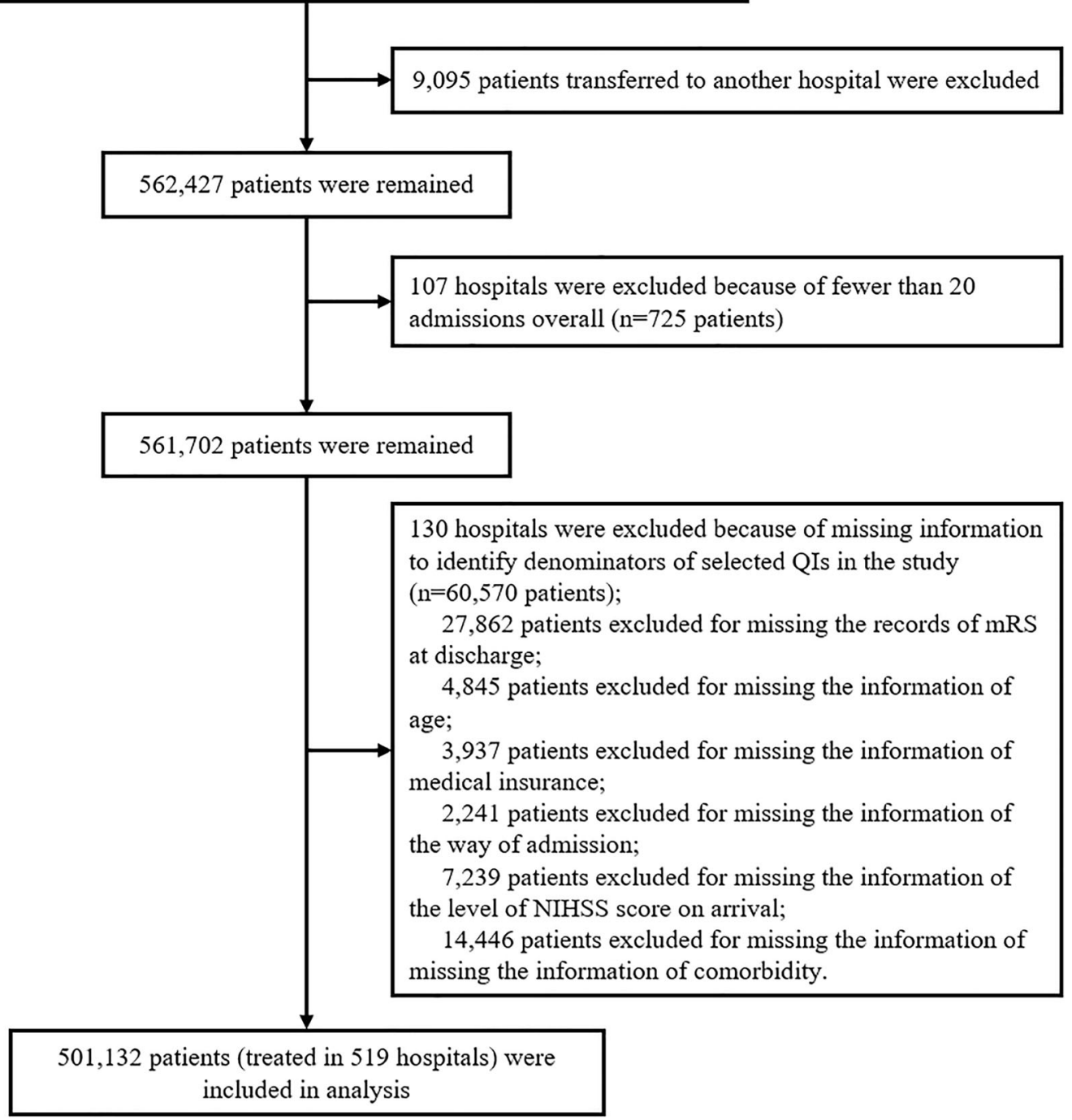

Figure 1 The flow chart of the selection of study participants. QI, quality indicator.

Cerebrovascular diseases included cerebral atherosclerosis, aneurysm, vascular malformation, and chronic cerebral circulation insufficiency. Hospital-level covariates included geographic region (east, midland, west), ownership (government owned, private owned), hospital-level (secondary, tertiary), university affiliated status, nurse-to-bed ratio $(\leq 0.4,0.4-0.6$, $0.6-0.8, \geq 0.8)$, medical personnel-to-bed ratio $(\leq 1.03,1.03$ $1.50,1.50-2.0, \geq 2.0)$, and annual outpatient volume.

\section{Statistical Analysis}

Patient and hospital baseline characteristics of the study population were described in counts and percentages. To assess performance on stroke care of the analytical population, adherence rate for each QI was presented as a proportion in which the sum of patients receiving care (numerator) was divided by the number of patients meeting the eligible definition (denominator). The hospital adherence rate of individual QIs was described in median and interquartile range.
For each indicator, the expected adherence rate was calculated from hierarchical logistic regression models fitted to the entire eligible patient population, adjusting relevant patientlevel covariates and setting the hospital level as random intercepts. Based on observed-to-expected $(\mathrm{O} / \mathrm{E})$ ratios and its statistical significance, we classified outlier hospitals performing better than expected as superior outliers, and those performing worse than expected as poor outliers. ${ }^{9}$ The performance status of hospital follows the binomial distribution; ${ }^{10,}{ }^{26}$ we used the exact binomial function to identify the significant $\mathrm{O} / \mathrm{E}$ outliers:

$P(m$ out of $n)=\frac{n !}{m !(n-m) !}\left(p^{m}\right)(1-p)^{n-m}$

where $n$ denotes the eligible patients for one indicator in a specific hospital, $m$ denotes the actual patients adhering to one indicator in a specific hospital, and $p$ denotes the model-based estimated adherence rate of one indicator for a specific hospital. ${ }^{10,18} \mathrm{Next}$, the hospitals were classified into five-level outliers using the following classification: (1) 
high outliers: $\mathrm{O} / \mathrm{E}>1$ and $P<0.05$; (2) at-risk high outlier: $\mathrm{O} /$ E $>1$ and $0.05<P<0.1$; (3) non-outlier: $\mathrm{O} / \mathrm{E}<1$ and $P>0.1$ and $\mathrm{O} / \mathrm{E}>1$ and $P>0.1$; (4) at-risk low outlier: $\mathrm{O} / \mathrm{E}<1$ and $0.05<P<0.1 ;$ (5) low outliers: $\mathrm{O} / \mathrm{E}<1$ and $P<0.05$. $\mathrm{O} / \mathrm{E}>1$ indicates that observed adherence of hospitals were higher than expected and $\mathrm{O} / \mathrm{E}<1$ indicates that observed adherence of hospitals were lower than expected. The five-level outliers were marked as $2,1,0,-1$, and -2 respectively. A test for trend of relationship between the outliers of hospital with and independent rate was conducted using the hierarchical logistic regression model with hospital outlier status as a continuous variable.

A composite quality measure that composite quality score (QS) was calculated encompassing hospital performance across the 13 indicators, for each indicator a hospital identified as one corresponding point, and the final QS was a summation of the points received across each indicator for an individual hospital. The association between a single indicator (high outlier vs low outlier), QS, and outcomes was investigated using a hierarchical logistic regression model. The correlation between QS and hospital characteristics was analyzed using the KruskalWallis $H$ test. The statistical analyses were performed with SAS software version 9.4 (SAS Institute, NC, USA) and a two-sided $P$ value of less than 0.05 was considered to indicate statistical significance.

There also have been numerous discussions about whether process measures should be adjusted for case mix. While outcomes comparisons traditionally accounted for differences in the patient case mix, process measures typically do not. ${ }^{19}$, ${ }^{20}$ Therefore, we also repeated the above analysis procedure, but did not adjust for case mix. We also calculated the QS by other two commonly used methods of numerator-based weights and "All-or-None," respectively. ${ }^{15,16}$ And we analyzed the relationships between different quality score and outcomes.

\section{RESULTS}

\section{Characteristics of Patients and Hospitals}

Table 1 shows the characteristics of patients and hospitals. The mean age of patients was $66.28 \pm 12.38$, $88.16 \%$ of patients were covered by medical insurance, $46.27 \%$ were admitted to hospital after emergency, and $44.40 \%$ were minor stroke (NIHSS $=1-4$ ). Diabetes mellitus, hematological disease, and cerebrovascular disease were the most common comorbidities. The mean length of stay was $12.93 \pm 7.71$ days. The mean health technician-to-bed ratio of all hospitals was $1.34 \pm 0.40$, and the mean nurse-to-bed ratio was $0.70 \pm 0.20$. More than half of the hospitals were in the eastern region, and almost all hospitals were tertiary and public. Meanwhile, $27.17 \%$ of patients were treated at university affiliated hospitals.
Table 1 Characteristics of Patients $(n=501,132)$ and Hospitals $(n=$ 519)

\begin{tabular}{|c|c|c|}
\hline Characteristic & $N$ & Percentage (\%) \\
\hline \multicolumn{3}{|l|}{ Age* } \\
\hline$\leq 55$ & 101,422 & 20.24 \\
\hline $55-65$ & 130,717 & 26.08 \\
\hline $65-75$ & 140,881 & 28.11 \\
\hline$>75$ & 128,112 & 25.56 \\
\hline \multicolumn{3}{|l|}{ Medical insurance* } \\
\hline Public medical insurance & 19,272 & 3.85 \\
\hline Basic medical insurance & 386,334 & 77.09 \\
\hline Commercial medical insurance & 36,217 & 7.23 \\
\hline Self-paying & 59,309 & 11.84 \\
\hline \multicolumn{3}{|l|}{ Way of admission* } \\
\hline Outpatient admission & 269,261 & 53.73 \\
\hline Emergency admission & 231,871 & 46.27 \\
\hline \multicolumn{3}{|l|}{ Level of NIHSS score on arrival* } \\
\hline No stroke symptoms & 42,518 & 8.48 \\
\hline Minor stroke & 222,496 & 44.40 \\
\hline Moderate stroke & 216,694 & 43.24 \\
\hline Moderate to severe stroke & 9787 & 1.95 \\
\hline Severe stroke & 9637 & 1.92 \\
\hline \multicolumn{3}{|l|}{ Comorbidity* } \\
\hline Diabetes mellitus & 27,403 & 5.47 \\
\hline Myocardial infarction & 2979 & 0.59 \\
\hline Pneumonia & 5343 & 1.07 \\
\hline Coronary heart disease & 11,529 & 2.30 \\
\hline Renal failure & 1940 & 0.39 \\
\hline Chronic obstructive pulmonary disease & 2875 & 0.57 \\
\hline Arrhythmia & 6145 & 1.23 \\
\hline Hematological diseases & 44,426 & 8.87 \\
\hline Cerebrovascular disease & 27,127 & 5.41 \\
\hline Rheumatic valvular heart disease & 2150 & 0.43 \\
\hline Congestive heart failure & 8177 & 1.63 \\
\hline \multicolumn{3}{|l|}{ Medical personnel-to-bed ratio ${ }^{\dagger}$} \\
\hline$\leq 1.03$ & 105 & 20.23 \\
\hline $1.03-1.50$ & 259 & 49.90 \\
\hline $1.50-2.0$ & 125 & 24.08 \\
\hline$>2.0$ & 30 & 5.78 \\
\hline \multicolumn{3}{|l|}{ Nurse-to-bed ratio ${ }^{\dagger}$} \\
\hline$\leq 0.40$ & 20 & 3.85 \\
\hline $0.40-0.60$ & 143 & 27.55 \\
\hline $0.60-0.80$ & 223 & 42.97 \\
\hline$>0.80$ & 133 & 25.63 \\
\hline \multicolumn{3}{|l|}{ Region $^{\dagger}$} \\
\hline East & 266 & 51.25 \\
\hline Midland & 99 & 19.08 \\
\hline West & 154 & 29.67 \\
\hline \multicolumn{3}{|l|}{ Ownership $^{\dagger}$} \\
\hline Private owned & 19 & 3.66 \\
\hline Government owned & 500 & 96.34 \\
\hline \multicolumn{3}{|l|}{ Hospital level $^{\dagger}$} \\
\hline Secondary & 19 & 3.66 \\
\hline Tertiary & 500 & 96.34 \\
\hline \multicolumn{3}{|l|}{ University affiliated status $^{\dagger}$} \\
\hline Non-affiliated hospital & 378 & 72.83 \\
\hline Affiliated hospital & 141 & 27.17 \\
\hline
\end{tabular}

*Patient characteristics

${ }^{\dagger}$ Hospital characteristics

\section{Adherence Rate of QIs}

The overall population eligible for each individual guideline indicator for acute ischemic stroke ranged from $3.49 \%$ for anticoagulant therapy (QI7) to $79.69 \%$ for dysphagia screening (QI11), except for two full eligible indicators (QI2 and QI9). Antiplatelet therapy (aspirin or clopidogrel) within $48 \mathrm{~h}$ of admission (QI8), evaluation of vascular function within 1 week of hospitalization (QI13), and statins therapy (QI10) occupied the top three adherence rates $(98.75 \%, 94.08 \%$, and $92.06 \%$, respectively). On the contrary, acute thrombolytic therapy of t-PA or urokinase (QI6) had the lowest rate, of only $22.88 \%$ (Table 2). 
Table 2 Adherence Rates of Quality Indicators and Hospital Variation

\begin{tabular}{|c|c|c|c|c|c|}
\hline Indicator & Eligible rate of patients (\%) & Denominator & Numerator & Overall adherence rate $(\%)$ & $\begin{array}{l}\text { Hospital variation median (IQR) } \\
(\%)\end{array}$ \\
\hline QI1 & 25.80 & 129,292 & 74,883 & 57.92 & $59.88(41.90-75.00)$ \\
\hline QI2 & 100.00 & 501,132 & 207,601 & 41.43 & $50.00(17.93-79.81)$ \\
\hline QI3 & 44.20 & 221,518 & 163,200 & 73.67 & $78.50(63.57-88.99)$ \\
\hline QI4 & 45.12 & 226,096 & 117,150 & 51.81 & $53.80(28.00-77.87)$ \\
\hline QI5 & 44.24 & 221,679 & 114,495 & 51.65 & $49.41(25.19-74.71)$ \\
\hline QI6 & 25.79 & 129,228 & 29,569 & 22.88 & $22.45(16.38-32.33)$ \\
\hline QI7 & 3.49 & 17,488 & 10,367 & 59.28 & $60.00(42.31-73.33)$ \\
\hline QI8 & 59.02 & 295,765 & 292,069 & 98.75 & $98.58(97.50-99.40)$ \\
\hline QI9 & 100.00 & 501,132 & 290,809 & 58.03 & $73.05(42.68-90.91)$ \\
\hline QI10 & 37.38 & 187,309 & 172,446 & 92.06 & $94.59(89.17-97.23)$ \\
\hline QI11 & 79.69 & 399,371 & 188,044 & 47.09 & $58.69(31.25-83.45)$ \\
\hline QI12 & 12.09 & 60,598 & 51,103 & 84.33 & $85.42(70.46-92.59)$ \\
\hline QI13 & 54.05 & 270,841 & 254,813 & 94.08 & $93.80(89.35-96.79)$ \\
\hline
\end{tabular}

QI quality indicator, IQR interquartile range

\section{QS}

For each QI, all hospitals were divided into five-level outliers according to observed-to-expected ratios and $P$ value. Table 3 shows the number of hospitals in each classification. From $0.39 \%$ (QI12) to $19.65 \%$ (QI5) of hospitals were identified as high outliers according to different QIs, except for QI7 and QI8, which did not identify any high outliers. For each QI, the higher level of hospital outlier status was associated with a significantly higher independent rate $(P$ for trend $<0.05)$, aside from the indicator QI8, QI10, and QI12. The QS ranged from -20 to 16. Distribution of QS was shown in Figure 2.

\section{Relationship Between Performance and Outcome}

The impact of quality variation on patient outcome was assessed using single indicators and composite QS (Fig. 3). After adjusting for patient and hospital characteristics, providers that were high outliers based on QI2, QI8, QI9, and QI11 exhibited better independence rates of treatment compared with low performance outliers, and odds ratios (ORs) were 2.660, 4.344, 4.114, and 3.345 , respectively. However, no significant difference in the independent rate was found between high and low outliers in terms of the other nine process indicators. For composite QS, each point increase predicted an $8 \%$ higher odds of independent rate $(\mathrm{OR}=1.080,95 \% \mathrm{CI}=1.057-1.105)$ (Table 4).

To better understand the possible structural elements driving quality variations, we evaluated associations between composite quality score and hospital characteristics (Supplement Figure 1). Hospitals with high QS had higher nurse-to-bed ratios and were more likely to be in eastern regions. However, these differences were not statistically significant.

\section{Sensitive Analysis}

The results without adjustment for case mix of the process measures were similar to the above analyses. The QS of hospital did not change much (see Supplement Figure 2). The adjusted OR of composite quality score from numeratorbased weights and "All-or-None" were similar to the equalweights method (see Supplement Table 2).

\section{DISCUSSION}

Maximizing the quality of care delivered is a promising target for government agencies and medical providers. Therefore, significant efforts have been made to establish hospital-level QIs guiding health policy decision-making, resource

Table 3 Number of 519 Hospitals Stratified by Outlier Classification

\begin{tabular}{|c|c|c|c|c|c|c|}
\hline \multirow[t]{2}{*}{ Indicator } & \multicolumn{5}{|c|}{ Number of hospitals stratified by outlier classification } & \multirow[t]{2}{*}{$P$ for trend } \\
\hline & Low outliers & At-risk low outlier & Non-outlier & At-risk high outlier & High outliers & \\
\hline QI1 & $71(13.68 \%)$ & $90(17.34 \%)$ & $216(41.62 \%)$ & $89(17.15 \%)$ & $53(10.21 \%)$ & $<0.0001$ \\
\hline Q12 & $131(25.24 \%)$ & $72(13.87 \%)$ & $130(25.05 \%)$ & $69(13.29 \%)$ & $117(22.54 \%)$ & $<0.0001$ \\
\hline QI3 & $116(22.35 \%)$ & $89(17.15 \%)$ & $178(34.30 \%)$ & $88(16.96 \%)$ & $48(9.25 \%)$ & 0.0032 \\
\hline QI4 & $91(17.53 \%)$ & $73(14.07 \%)$ & $176(33.91 \%)$ & $80(15.41 \%)$ & $99(19.08 \%)$ & 0.0004 \\
\hline QI5 & $84(16.18 \%)$ & $84(16.18 \%)$ & $172(33.14 \%)$ & $77(14.84 \%)$ & $102(19.65 \%)$ & 0.0007 \\
\hline QI6 & $60(11.56 \%)$ & $94(18.11 \%)$ & $207(39.88 \%)$ & $97(18.69 \%)$ & $61(11.75 \%)$ & $<0.0001$ \\
\hline QI7 & $2(0.38 \%)$ & $39(7.51 \%)$ & $455(87.67 \%)$ & $23(4.43 \%)$ & $0(0.00 \%)$ & 0.0269 \\
\hline QI8 & $4(0.77 \%)$ & $59(11.37 \%)$ & $450(86.70 \%)$ & $6(1.16 \%)$ & $0(0.00 \%)$ & 0.0693 \\
\hline QI9 & $168(32.37 \%)$ & $65(12.52 \%)$ & $120(23.12 \%)$ & $83(15.99 \%)$ & $83(15.99 \%)$ & $<0.0001$ \\
\hline QI10 & $39(7.51 \%)$ & $93(17.92 \%)$ & $330(63.58 \%)$ & $53(10.21 \%)$ & $4(0.77 \%)$ & 0.1640 \\
\hline QI11 & $146(28.13 \%)$ & $66(12.72 \%)$ & $134(25.82 \%)$ & $87(16.76 \%)$ & $86(16.57 \%)$ & $<0.0001$ \\
\hline QI12 & $12(2.31 \%)$ & $86(16.57 \%)$ & $401(77.26 \%)$ & $18(3.47 \%)$ & $2(0.39 \%)$ & 0.7316 \\
\hline QI13 & $44(8.48 \%)$ & $102(19.65 \%)$ & $274(52.79 \%)$ & $91(17.53 \%)$ & $8(1.54 \%)$ & 0.0046 \\
\hline
\end{tabular}

QI quality indicator 


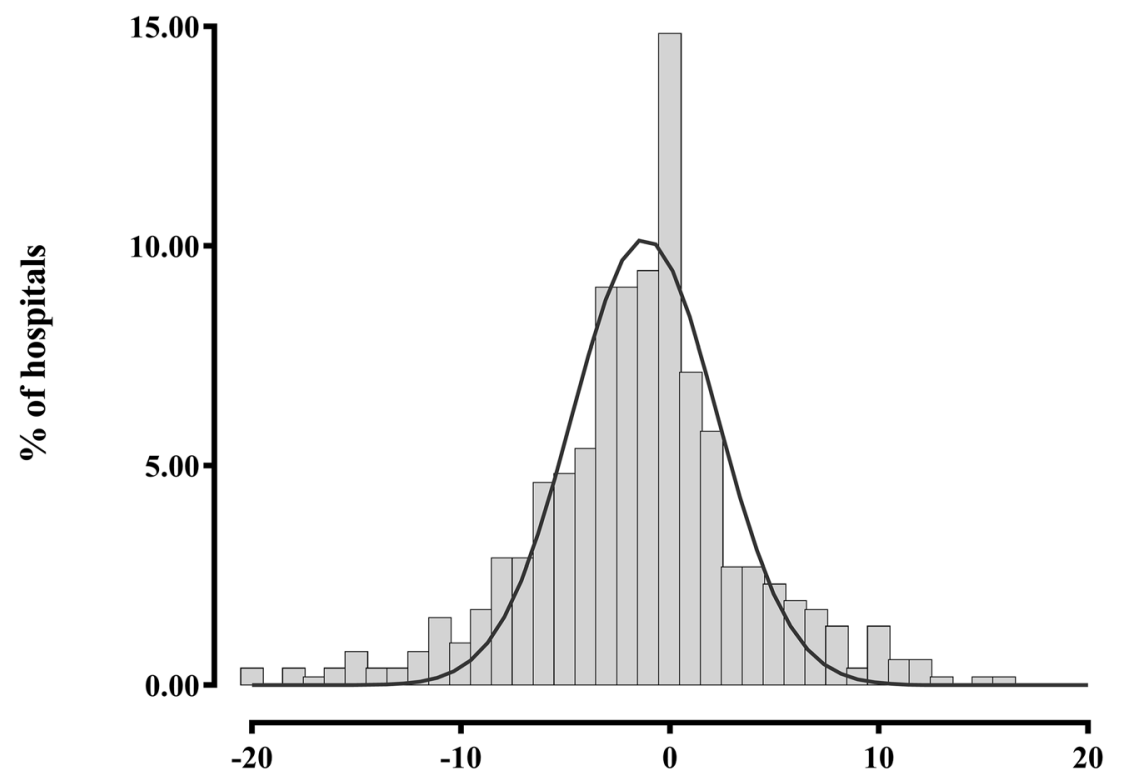

\section{Quality score}

Figure 2 Distribution of quality score (QS) among hospitals.

allocation, educational initiatives, and patient choice. Moreover, accurately identifying the degree of quality variations and the impact of this variation on patient outcomes according to validated QIs is an important issue. In the current study, we developed a risk-adjusted quality score according to process indicators to benchmark the quality of acute stroke care at the hospital level. Utilizing this tool, we revealed wide variation in the quality of care that patients received, and demonstrated the positive consequences on important patient outcomes of reducing this variation.

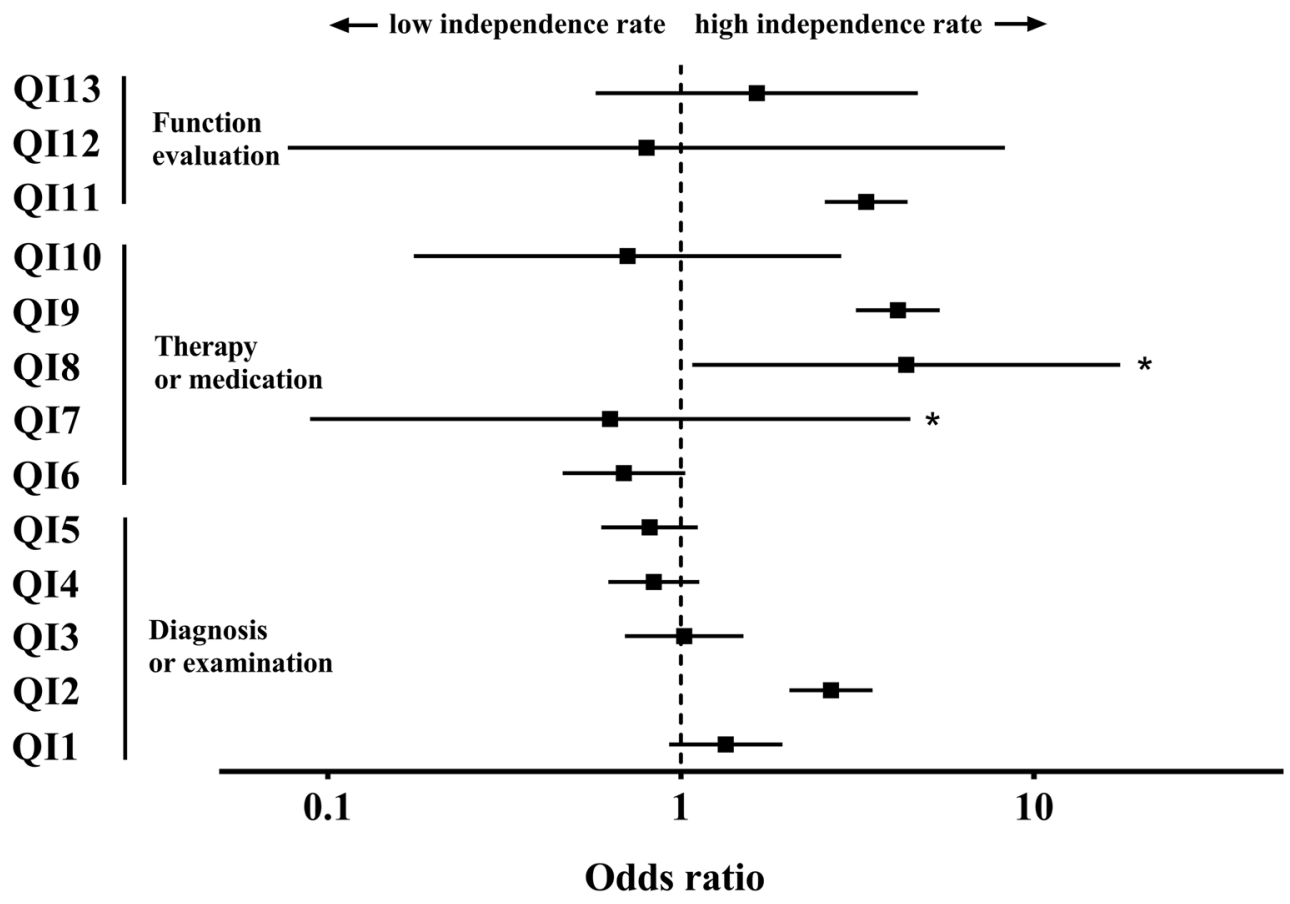

Figure 3 Adjusted association between hospital outlier status (high outlier versus low performance outlier) and patient outcome according to single and composite quality indicators. *The ORs of QI7 and QI8 were calculated by at-risk high outlier vs low outlier because there was no hospital identified as high outlier based on these two indicators. 
Table 4 Predictors of Independence Rate by Multivariable Analysis

\begin{tabular}{|c|c|c|}
\hline Characteristic & OR & $95 \% \mathrm{CL}$ \\
\hline \multicolumn{3}{|l|}{ Age* (ref = "> 75") } \\
\hline$\leq 55$ & 1.141 & $1.117-1.166$ \\
\hline $55-65$ & 1.106 & $1.085-1.129$ \\
\hline $65-75$ & 1.112 & $1.091-1.134$ \\
\hline \multicolumn{3}{|l|}{ Medical insurance* (ref = "self-paying") } \\
\hline Public medical insurance & 1.09 & $1.065-1.115$ \\
\hline Basic medical insurance & 1.058 & $1.022-1.095$ \\
\hline Commercial medical insurance & 0.705 & $0.675-0.737$ \\
\hline \multicolumn{3}{|c|}{ Way of admission* (ref = "emergency admission") } \\
\hline Outpatient admission & 0.943 & $0.929-0.958$ \\
\hline \multicolumn{3}{|c|}{ Level of NIHSS score on arrival* (ref="0") } \\
\hline 1 & 0.822 & $0.761-0.888$ \\
\hline 2 & 0.824 & $0.774-0.877$ \\
\hline 3 & 0.902 & $0.848-0.96$ \\
\hline 4 & 0.948 & $0.872-1.03$ \\
\hline \multicolumn{3}{|l|}{ Comorbidity* } \\
\hline Diabetes mellitus & 2.935 & $2.807-3.069$ \\
\hline Myocardial infarction & 1.549 & $1.349-1.779$ \\
\hline Pneumonia & 0.968 & $0.887-1.055$ \\
\hline Coronary heart disease & 2.111 & $1.968-2.263$ \\
\hline Renal failure & 0.907 & $0.78-1.054$ \\
\hline Chronic obstructive pulmonary disease & 1.644 & $1.433-1.886$ \\
\hline Arrhythmia & 1.326 & $1.22-1.442$ \\
\hline Hematological diseases & 3.461 & $3.321-3.607$ \\
\hline Cerebrovascular disease & 3.045 & $2.901-3.197$ \\
\hline Rheumatic valvular heart disease & 2.42 & $2.073-2.824$ \\
\hline \multirow{2}{*}{\multicolumn{3}{|c|}{ Health technician-to-bed ratio ${ }^{\dagger}($ ref $=" \leq 1.03 ")$}} \\
\hline & & \\
\hline $1.03-1.50$ & 1.48 & $0.971-2.256$ \\
\hline $1.50-2.0$ & 1.614 & $0.865-3.011$ \\
\hline$>2.0$ & 0.943 & $0.415-2.143$ \\
\hline \multicolumn{3}{|l|}{ Nurse-to-bed ratio ${ }^{\dagger}($ ref $=$ “ $\leq 0.40 ")$} \\
\hline $0.40-0.60$ & 0.745 & $0.385-1.445$ \\
\hline $0.60-0.80$ & 0.632 & $0.3-1.328$ \\
\hline$>0.80$ & 0.626 & $0.261-1.501$ \\
\hline \multicolumn{3}{|l|}{ Region $^{\dagger}$ (ref = "west") } \\
\hline East & 0.812 & $0.611-1.078$ \\
\hline Midland & 0.683 & $0.484-0.963$ \\
\hline \multicolumn{3}{|l|}{ Ownership ${ }^{\dagger}$ (ref = "private owned") } \\
\hline Government owned & 1.285 & $0.678-2.436$ \\
\hline \multicolumn{2}{|l|}{ Hospital level" (ref = "secondary") } & \\
\hline \multirow{2}{*}{\multicolumn{3}{|c|}{ University affiliated status ${ }^{\dagger}$ (ref $=$ "non-affiliated hospital") }} \\
\hline & & \\
\hline Affiliated hospital & $0.999^{1}$ & $0.998-1.001$ \\
\hline Composite quality score & 1.080 & $1.057-1.105$ \\
\hline
\end{tabular}

In the USA and other countries, there were many articles comparing hospital performance of stroke care based on process and outcome indicators. ${ }^{2,8,13,15,16}$ However, no related studies of identifying performance outliers for stroke care in Chinese population have been carried on thus far. Outcomebased indicators take a longer time to acquire, are more expensive to gather, and may have a very low incidence. ${ }^{11}$ From the database used in the current study, we collected the mRS scores as in-hospital outcomes, in which patient who died in hospital $(\mathrm{mRS}=6)$ accounted for only $0.56 \%$. Comparing with prior research on quality of care for stroke based on outcomes measured using the Rankin Score, ${ }^{21-23}$ because of the low incidence rate of outcomes, it may be not adequate risk adjustment that accurately reflects the quality of care a patient actually received. In our study, 13 process indicators covering examination, functional assessment, and medication were examined to assess the quality of acute stroke care. This approach was similar to that used in many previous studies, but with a wider range of indicators. ${ }^{27-30}$ Process indicators can be measured relatively early in the spectrum of care, giving providers a chance to understand their performance and adjust their internal processes to benefit patients entering these hospitals for treatment in future. ${ }^{10}$ Literatures indicate that it is more reasonable to use independence to evaluate the prognosis of patients as stroke patients have a high disability rate. ${ }^{7,22}$ Thus, we used the independence (mRS $\left.=0-2\right)$ as inhospital outcomes and developed a further composite measure of acute stroke quality incorporating all indicators analyzed in this study to resolve the problem of poor consistency of the relationships between different process indicators and outcomes. Moreover, we verified that the QS values were associated with patient outcomes, consistent with the assumptions of the Donabedian model.

The current finding that better health outcomes could be achieved by improving performance in acute stroke care suggests that many undesired outcomes (i.e., dependence or died) could be avoided by implementing clinical practice guidelines and improving patients' adherence to treatment recommendations. This finding also confirmed the practicality and validity of the currently proposed quality score, identifying and improving performance of acute stroke care.

The current study involved several limitations that should be considered. First, although we used a nationwide population-based database, some confounding variables were likely missed. Therefore, these variables may not be included in the multilevel model. Second, we were unable to determine the contribution of better adherence to QIs on long-term outcomes because of the absence of follow-up data. Third, we measured outcome of stroke patients using independence at discharge $(m R S=0-2)$, but it can only partly explain the quality of stroke care. Fourth, equal-weights is the most common method to create composite QS, and results were similar to other methods. However, the association of different stroke process measures on outcomes may be different. Finally, the results were heavily influenced by the Chinese healthcare infrastructure and health system. Thus, these findings may not be generalizable to other countries. Similar quality assessments utilizing population-level databases outside China will be required to confirm the external validity of our results.

\section{CONCLUSION}

In China, nationwide variation in the quality of acute stroke care exists at the hospital level. The current results revealed that these variations could be captured by the quality score we constructed, providing a validated specific composite measure of quality. Specifically, we found that patients were likely to receive better outcomes if they received treatment in high outlier hospitals. Our acute stroke quality score may be a 
useful quality benchmarking tool for acute stroke care, which can provide audit-level feedback to policymakers and hospitals for quality improvement.

Corresponding Author: Meina Liu, MD; Department of Biostatistics, Public Health College, Harbin Medical University Harbin, China (e-mail: liumeina369@163.com).

Funding Information: This work was supported by the National Natural Science Foundation of China (Grant Number 81573255 to Meina Liu), which participated in the design of the study and data collection.

\section{Compliance with Ethical Standards:}

Conflict of Interest: The authors declare that they do not have a conflict of interest.

\section{REFERENCES}

1. Feigin VL, Mensah GA, Norrving B, Murray CJ, Roth GA. Atlas of the global burden of stroke (1990-2013): the GBD 2013 study. Neuroepidemiology. 2015;45(3):230-6.

2. Wiedmann S, Hillmann $\mathbf{S}$, Abilleira $\mathbf{S}$, et al. Variations in acute hospital stroke care and factors influencing adherence to quality indicators in 6 European audits. Stroke. 2015;46(2):579-81.

3. Wang $\mathbf{Y}$, Liao $\mathbf{X}$, Zhao $\mathbf{X}$, et al. Using recombinant tissue plasminogen activator to treat acute ischemic stroke in China: analysis of the results from the Chinese National Stroke Registry (CNSR). Stroke. 2011;42(6):1658-64.

4. Normand S-LT, Glickman ME, Gatsonis CA. Statistical methods for profiling providers of medical care: issues and applications. Journal of the American Statistical Association. 1997;92(439):803-14.

5. Rubin HR, Pronovost P, Diette GB. Methodology Matters. From a process of care to a measure: the development and testing of a quality indicator. International Journal for Quality in Health Care. 2001;13(6):489-96.

6. Pérez A, Dennis RJ, Rondón MA, Metcalfe MA, Rowan KM. A Colombian survey found intensive care mortality ratios were better in private vs. public hospitals. Journal of clinical epidemiology. 2006;59(1):94-101.

7. Katzan IL, Spertus J, Bettger JP, et al. Risk Adjustment of Ischemic Stroke Outcomes for Comparing Hospital Performance: A Statement for Healthcare Professionals From the American Heart Association/ American Stroke Association. Stroke. 2014;45(3):918-44.

8. Shahian DM, Normand S-LT. Comparison of "Risk-Adjusted" Hospital Outcomes. Circulation. 2008;117(15):1955-63.

9. Best WR, Cowper DC. The ratio of observed-to-expected mortality as a quality of care indicator in non-surgical VA patients. Medical care. 1994;32(4):390-400

10. Massarweh NN, Hu C-Y, You YN, et al. Risk-adjusted pathologic margin positivity rate as a quality indicator in rectal cancer surgery. Journal of Clinical Oncology. 2014;32(27):2967.

11. Dimick JB, Welch HG, Birkmeyer JD. Surgical mortality as an indicator of hospital quality: the problem with small sample size. Jama. 2004;292(7):847-51.

12. Birkmeyer JD, Dimick JB, Birkmeyer NJ. Measuring the quality of surgical care: structure, process, or outcomes? Journal of the American College of Surgeons. 2004;198(4):626-32.
13. Ingeman A, Pedersen L, Hundborg HH, et al. Quality of care and mortality among patients with stroke: a nationwide follow-up study. Medical care. 2008;46(1):63-9.

14. Kruk ME, Gage AD, Joseph NT, Danaei G, Garcia-Saisó S, Salomon JA. Mortality due to low-quality health systems in the universal health coverage era: a systematic analysis of amenable deaths in 137 countries. The Lancet. 2018;392(10160):2203-12.

15. Couralet M, Guérin S, Le Vaillant M, Loirat $\mathbf{P}$, Minvielle E. Constructing a composite quality score for the care of acute myocardial infarction patients at discharge: impact on hospital ranking. Medical care. 2011:569-76.

16. Peterson ED, DeLong ER, Masoudi FA, et al. ACCF/AHA 2010 position statement on composite measures for healthcare performance assessment: American College of Cardiology Foundation/American Heart Association Task Force on performance measures (writing committee to develop a position statement on composite measures). Journal of the American College of Cardiology. 2010;55(16):1755-66

17. Schroeck FR, Kaufman SR, Jacobs BL, et al. Adherence to Performance Measures and Outcomes among Men Treated for Prostate Cancer. Journal of Urology. 192(3):743-8.

18. Lawson KA, Saarela O, Abouassaly R, Kim SP, Breau RH, Finelli A. The impact of quality variations on patients undergoing surgery for renal cell carcinoma: a National Cancer Database study. European urology. 2017;72(3):379-86

19. Mehta RH, Liang $\mathbf{L}$, Karve AM, et al. Association of patient case-mix adjustment, hospital process performance rankings, and eligibility for financial incentives. Jama. 2008;300(16):1897-903.

20. Paddison C, Elliott M, Parker R, et al. Should measures of patient experience in primary care be adjusted for case mix? Evidence from the English General Practice Patient Survey. BMJ Qual Saf. 2012;21(8):63440 .

21. Kasner SE. Clinical interpretation and use of stroke scales. The Lancet Neurology. 2006;5(7):603-12.

22. Saver JL, Filip B, Hamilton S, et al. Improving the reliability of stroke disability grading in clinical trials and clinical practice: the Rankin Focused Assessment (RFA). Stroke. 2010;41(5):992-5.

23. Zhang B, Gao $\mathbf{C}$, Yang $\mathbf{N}$, et al. Is elevated SUA associated with a worse outcome in young Chinese patients with acute cerebral ischemic stroke? BMC neurology. 2010;10(1):82.

24. Saposnik G, Cote R, Mamdani M, et al. JURaSSiC: accuracy of clinician vs risk score prediction of ischemic stroke outcomes. Neurology. 2013;81(5):448-55.

25. Hage V. The NIH stroke scale: a window into neurological status. NurseCom Nursing Spectrum (Greater Chicago). 2011;24(15):44-9.

26. Russell MC, You YN, Hu C-Y, et al. A novel risk-adjusted nomogram for rectal cancer surgery outcomes. JAMA surgery. 2013;148(8):769-77.

27. Roychoudhury C, Jacobs BS, Baker PL, Schultz D, Mehta RH, Levine SR. Acute ischemic stroke in hospitalized medicare patients: evaluation and treatment. Stroke. 2004;35(1):e22-e3.

28. Sposato LA, Esnaola MaM, Zamora R, et al. Quality of ischemic stroke care in emerging countries: the Argentinian National Stroke Registry (ReNACer). Stroke. 2008;39(11):3036-41.

29. Panella M, Marchisio S, Brambilla R, Vanhaecht K, Di Stanislao F. A cluster randomized trial to assess the effect of clinical pathways for patients with stroke: results of the clinical pathways for effective and appropriate care study. BMC medicine. 2012;10(1):71.

30. Hong-Rong $\mathbf{Y}$, Lei M, Yi-Feng JIANG Y-CW, Eugene $\mathbf{C}$, Yan-Hong $Z$. Process and Results of Implementing Disease Management Program in Patients with First-time Ischemic Stroke. Iranian journal of public health. 2018;47(7): 1007

Publisher's Note Springer Nature remains neutral with regard to jurisdictional claims in published maps and institutional affiliations. 